ELECTRONIC RESEARCH ANNOUNCEMENTS OF THE AMERICAN MATHEMATICAL SOCIETY

Volume 11, Pages 21-33 (March 7, 2005)

S $1079-6762(05) 00143-5$

\title{
WAVELET CONSTRUCTIONS IN NON-LINEAR DYNAMICS
}

\author{
DORIN ERVIN DUTKAY AND PALLE E.T. JORGENSEN
}

(Communicated by Boris Hasselblatt)

\begin{abstract}
We construct certain Hilbert spaces associated with a class of nonlinear dynamical systems $X$. These are systems which arise from a generalized self-similarity and an iterated substitution. We show that when a weight function $W$ on $X$ is given, then we may construct associated Hilbert spaces $H(W)$ of $L^{2}$-martingales which have wavelet bases.
\end{abstract}

\section{INTRODUCTION}

A particularly productive approach to the construction of wavelet bases in $L^{2}(\mathbb{R})$ is based on the notion from optics of resolution, which translates into scales of nested Hilbert subspaces $V_{n}, n \in \mathbb{Z}$, in $L^{2}(\mathbb{R})$ such that the intersection is $\{0\}$ and the union is dense. Moreover the operation of dyadic scaling transforms each $V_{n}$ to the next $V_{n+1}$. This is called a multiresolution approach to wavelets (see [Dau92]), and it is based on the interplay between the two abelian groups $\mathbb{T}$ (the circle group $=$ onetorus), and $\mathbb{R}$ (the real line), with $\mathbb{T}$ representing a period interval placed on the line $\mathbb{R}$. This paper is based on the observation that multiresolutions really are martingales; and by exploiting this fact, we are able to adapt the geometric idea of subspaces $\left(V_{n}\right)$ to nonlinear dynamics in a variety of applications where such a pair of groups is not available, but instead there is a single endomorphism on a compact space $X$ which defines a certain self-similarity mirroring the more familiar scale-similarity that is so powerful in wavelet analysis.

The words 'non-linear' and 'wavelets' in the title beg two questions: (1) "What is the Hilbert space?" (2) If 'non-linear', then there must be a substitute for the duality between the operators of translation and multiplication!?

We will address the questions in the announcement below, while giving answers with full proofs in the forthcoming papers DJ03, DJ04a, DJ04b, DJ04c.

We have in mind three classes of examples: (1) The state space $X$ for a subshift system in symbolic dynamics; (2) affine iterated function systems based on a fixed expansive scaling matrix; and (3) the complex iteration systems which generate Julia sets $X$ in the Riemann sphere. If $r(z)$ is a rational function, set

Received by the editors October 28, 2004.

2000 Mathematics Subject Classification. Primary 60G18; Secondary 42C40, 46G15, 42A65, 28A50, 30D05, 47D07, 37F20.

Key words and phrases. Measures, projective limits, transfer operator, martingale, fixed point, multiresolution, Julia set, subshift, wavelet.

(C)2005 American Mathematical Society Reverts to public domain 28 years from publication 
$r^{n}=\underbrace{r \circ \cdots \circ r}_{n \text { times }}$. Then the Julia set $X=X(r)$ is the complement of the largest open subset of $\mathbb{C}$ where $r^{n}$ is a normal family.

The first step to construct a Hilbert space in any of these three classes of examples is the identification of the appropriate covariant measures on $X$, and the second step is the construction of a certain lifting from $X$ to a suitable space $X_{\infty}$ of discrete paths which originate in $X$. Specifically, $X_{\infty}:=\left\{\left(x_{n}\right) \in X^{\mathbb{N}} \mid r\left(x_{n+1}\right)=x_{n}, n \in\right.$ $\mathbb{N}\}$. We begin (in Section 21) by recalling how the familiar wavelet construction may be obtained from $X_{\infty}$ in the special case when $X=\mathbb{T}=\{z \in \mathbb{C}|| z \mid=1\}$, and $r(z)=z^{2}$. While the real line $\mathbb{R}$ is not $\mathbb{T}_{\infty}$, we may still build the Hilbert space $L^{2}(\mathbb{R})$ with its multiresolution wavelets from an inductive and isometric procedure based on $\mathbb{T}_{\infty}$. The intuitive idea is to get $\mathbb{R}$ in the limit by successive doubling of periods. But we use Hilbert spaces, and the idea is outlined in (2.5). It is further based on martingales. To highlight the distinction between the compact group $G=\mathbb{T}_{\infty}$ (called a solenoid) and the reals $\mathbb{R}$, contrast the following two familiar short exact sequences of abelian groups: $0 \rightarrow C \rightarrow G \rightarrow \mathbb{T} \rightarrow 0$, where $C$ is the Cantor group, on the one hand; and $0 \rightarrow \mathbb{Z} \hookrightarrow \mathbb{R} \rightarrow \mathbb{T} \rightarrow 0$, on the other. So while the groups $G$ and $\mathbb{R}$ are very different, the use of $G$ helps us build the Hilbert space $L^{2}(\mathbb{R})$, but within the category of isometries in Hilbert space. Moreover, the $X_{\infty}$ viewpoint is useful in important applications outside the group context.

We now introduce weight functions which are determined from our candidate for low-pass filters $m$. In our analysis, a low-pass filter will be a function $m$ on $X$ which satisfies axioms that generalize those which are known for standard wavelets in $L^{2}(\mathbb{R})$. Similarly the relation between the circle $\mathbb{T}$, and the real line $\mathbb{R}$, for familiar wavelets is mirrored in our lifting from $X$ to the space $X_{\infty}$.

A second feature in our analysis is a certain Perron-Frobenius-Ruelle operator $R=R_{W}$ associated with a non-negative weight function $W$ which may be taken to be the absolute value-square of $m$.

In addressing the second question, we give up the analogue of translations, but for the case of Julia sets $X$, we work instead with the operator of multiplication by the variable $z$ restricted to $X$. Our Hilbert space will be a Hilbert space built on martingales on $X_{\infty}$, and the unitary operator $U$ which corresponds to the familiar dyadic, or $N$-adic scaling for familiar wavelets will simply be the substitution of our system. This operator $U$ will scale between levels of our discrete $L^{2}$-martingales. Hence we arrive at a class of generalized multiresolutions, or multiwavelets. In this setting, we are able to prove a version of the dimension consistency formula of Baggett-Merrill et al. [BaM99, and to construct our wavelets.

A new feature of our analysis is a dichotomy between properties of our low-pass filters: The low/high-pass conditions in our context will refer to either a finite set of points or to a singular measure with full support. In the case of the middle-third Cantor set, this measure will be a classical infinite product Riesz measure.

What the non-linear settings have in common with the classical linear cases (e.g., wavelets) is a certain dichotomy. The non-linear systems we consider carry a certain strongly invariant measure. The support of this invariant measure must either be full, or it must be finite. In the case when the support is full we can expect the same rigidity as is known in familiar wavelet constructions, namely that the filters giving wavelets in the same space have equal absolute value. When the support is finite, we construct some scaling function as an infinite product, and we show 
that our generalized "low-pass" condition implies the existence of a wavelet basis construction is in a fixed Hilbert space, avoiding redundant multiplicity. We offer results on the nature of the Hilbert space which carries wavelet bases for both cases in our dichotomy: full support, and finite support. In either case, our construction uses a family of discrete time $L^{2}$-martingales. We establish a geometric setting which does not depend on the generalized wavelet filter. We then give conditions on two filters which imply isomorphism of the corresponding wavelet systems when the support of our invariant measure is some given finite cycle.

In the case of wavelets, [BDP04], it is $L^{2}(\mathbb{R})$, or a finite direct sum of $L^{2}(\mathbb{R})$ with itself. In that case the complex function is $z \rightarrow z^{N}$, and the Julia set is the circle $\mathbb{T}$.

Due to results of Bramson and Kalikow BK93 the strongly invariant measure may be non-unique, even in the case of a full shift, if the weight $W$ is just continuous.

\section{LINEAR VS NON-LINEAR: MARTINGALES}

In this paper we study the problem of inducing operators on Hilbert space from non-invertible transformations on compact metric spaces. The operators, or representations must satisfy relations which mirror properties of the given point transformations.

While our setup allows a rather general formulation in the context of $C^{*}$-algebras, we will emphasize the case of induction from an abelian $C^{*}$-algebra. Hence, we will stress the special case when $X$ is a given compact metric space, and $r: X \rightarrow X$ is a finite-to-one mapping of $X$ onto $X$. Several of our results are in the measurable category; and in particular we are not assuming continuity of $r$, or any contractivity properties.

If $r: X \rightarrow X$ (onto) is given, we let $X_{\infty}$ denote the projective limit

$$
X \stackrel{r}{\longleftarrow} X \stackrel{r}{\longleftarrow} X \longleftarrow X_{\infty}
$$

i.e., $X_{\infty}:=\left\{\left(x_{n}\right)_{n \in \mathbb{N}_{0}} \mid r\left(x_{n+1}\right)=x_{n}\right\}$. Then $r$ extends to an automorphism $\hat{r}$ determined by

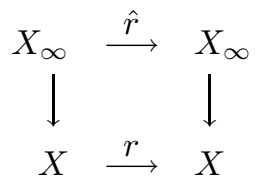

2.1. Wavelets. Our results will apply to wavelets. In the theory of multiresolution wavelets, the problem is to construct a special basis in the Hilbert space $L^{2}\left(\mathbb{R}^{d}\right)$ from a set of numbers $a_{n}, n \in \mathbb{Z}^{d}$.

The starting point is the scaling identity

$$
\varphi(t)=N^{1 / 2} \sum_{n \in \mathbb{Z}^{d}} a_{n} \varphi(A t-n) \quad\left(t \in \mathbb{R}^{d}\right),
$$

where $A$ is a $d$ by $d$ matrix over $\mathbb{Z}$, with eigenvalues $|\lambda|>1$, and $N=|\operatorname{det} A|$, and where $\varphi$ is a function in $L^{2}\left(\mathbb{R}^{d}\right)$.

The first problem is to determine when (2.2) has a solution in $L^{2}\left(\mathbb{R}^{d}\right)$, and to establish how these solutions (scaling functions) depend on the coefficients $a_{n}$.

When the Fourier transform is applied, we get the equivalent formulation,

$$
\hat{\varphi}(x)=N^{-1 / 2} m_{0}\left(A^{t r-1} x\right) \hat{\varphi}\left(A^{t r-1} x\right),
$$


where $\hat{\varphi}$ denotes the Fourier transform,

$$
\hat{\varphi}(x)=\int_{\mathbb{R}^{d}} e^{-i 2 \pi x \cdot t} \varphi(t) d t,
$$

and where now $m_{0}$ is a function on the torus

$$
\mathbb{T}^{d}=\left\{z=\left(z_{1}, \ldots, z_{d}\right) \in \mathbb{C}^{d}|| z_{j} \mid=1,1 \leq j \leq d\right\}=\mathbb{R}^{d} / \mathbb{Z}^{d},
$$

i.e., $m_{0}(z)=\sum_{n \in \mathbb{Z}^{d}} a_{n} z^{n}=\sum_{n \in \mathbb{Z}^{d}} a_{n} e^{-i 2 \pi n \cdot x}\left(z=e^{-2 \pi i \cdot x}\right)$. The duality between the compact group $\mathbb{T}^{d}$ and the lattice $\mathbb{Z}^{d}$ is given by $\langle z \mid n\rangle=z^{n}=z_{1}^{n_{1}} \cdots z_{d}^{n_{d}}$, $z=\left(z_{1}, \ldots, z_{d}\right), n=\left(n_{1}, \ldots, n_{d}\right)$.

In this case, matrix multiplication $x \mapsto A x$ on $\mathbb{R}^{d}$ passes to the quotient $\mathbb{R}^{d} / \mathbb{Z}^{d}$, and we get an $N$-to-one mapping $x \mapsto A x \bmod \mathbb{Z}^{d}$, which we denote by $r_{A}$.

The function $m_{0}$ is called a low pass filter, and it is chosen such that the operator $S=S_{m_{0}}$ given by $(S f)(z)=m_{0}(z) f(A z)$ is an isometry on $H_{0}=L^{2}\left(\mathbb{T}^{d}\right.$, Haar measure). Moreover, $L^{\infty}\left(\mathbb{T}^{d}\right)$ acts as multiplication operators on $H_{0}$. If $g \in L^{\infty}(\mathbb{T})$, then $(M(g) f)(z)=g(z) f(z)$, and

$$
S M(g)=M(g(A \cdot)) S .
$$

A main problem is the extension of this covariance relation (2.4) to a bigger Hilbert space $H_{0} \rightarrow H_{\text {ext }}, S \rightarrow S_{\text {ext }}$, such that $S_{\text {ext }}$ is unitary in $H_{\text {ext }}$. We now sketch briefly this extension in some concrete cases of interest.

We construct a sequence of measures $\omega_{0}, \omega_{1}, \ldots$ on $\mathbb{T}^{d}$ such that $L^{2}\left(\mathbb{T}^{d}, \omega_{0}\right) \simeq H_{0}$, and such that there are natural isometric embeddings

$$
L^{2}\left(\mathbb{T}^{d}, \omega_{n}\right) \hookrightarrow L^{2}\left(\mathbb{T}^{d}, \omega_{n+1}\right), \quad f \mapsto f \circ r_{A} .
$$

The limit in (2.5) defines a martingale Hilbert space $\mathcal{H}$ in such a way that the norm of the $L^{2}$-martingale $f$ is $\|f\|^{2}=\lim _{n \rightarrow \infty}\left\|P_{n} f\right\|_{L^{2}\left(\mathbb{T}^{d}, \omega_{n}\right)}^{2}$. In [DJ04a], we also prove an associated pointwise a.e. convergence theorem.

If $\Psi: L^{2}\left(\mathbb{T}^{d}, \omega_{n}\right) \rightarrow L^{2}\left(\mathbb{R}^{d}\right)$ is defined by $\Psi: f_{n} \mapsto f_{n}\left(A^{-n} x\right) \hat{\varphi}(x)$, then $\Psi$ is an isometry of $L^{2}\left(\mathbb{T}^{d}, \omega_{n}\right)$ into $L^{2}\left(\mathbb{R}^{d}\right)$.

Specifically,

$$
\int_{\mathbb{T}^{d}}\left|f_{n}\right|^{2} d \omega_{n}=\int_{\mathbb{R}^{d}}\left|f_{n}\left(A^{-n} x\right) \hat{\varphi}(x)\right|^{2} d x .
$$

As a result we have induced a system $\left(r_{A}, \mathbb{T}^{d}\right) \rightarrow\left(S_{m_{0}}, L^{2}\left(\mathbb{T}^{d}\right)\right) \rightarrow\left(U_{A}, L^{2}\left(\mathbb{R}^{d}\right)\right)$ where

$$
\left(U_{A} f\right)(x)=N^{1 / 2} f(A x) \quad\left(f \in L^{2}\left(\mathbb{R}^{d}\right), U_{A} \text { unitary }\right) ;
$$

the system is determined by the given filter function $m_{0}$. It can be checked that $\Psi$ is an isometry, and that $U_{A} M(g)=M(g(A \cdot)) U_{A}$ holds on $L^{2}\left(\mathbb{R}^{d}\right)$. Moreover $\Psi$ maps onto $L^{2}\left(\mathbb{R}^{d}\right)$ if the function $m_{0}$ does not vanish on a subset of positive measure.

In the case of wavelets, we ask for a wavelet basis in $L^{2}\left(\mathbb{R}^{d}\right)$ which is consistent with a suitable resolution subspace in $L^{2}\left(\mathbb{R}^{d}\right)$. Whether the basis is orthonormal, or just a Parseval frame, it may be constructed from a system of subband filters $m_{i}$, say with $N$ frequency bands. These filters $m_{i}$ may be realized as functions on $X=\mathbb{T}^{d}=\mathbb{R}^{d} / \mathbb{Z}^{d}$, the $d$-torus. Typically the scaling operation is specified by a given expansive integral $d$ by $d$ matrix $A$. 
Let $N:=|\operatorname{det} A|$. Pass $A$ to the quotient $X=\mathbb{R}^{d} / \mathbb{Z}^{d}$, and we get a mapping $r$ of $X$ onto $X$ such that $\# r^{-1}(x)=N$ for all $x$ in $X$, and the $N$ branches of the inverse are strictly contractive in $X=\mathbb{R}^{d} / \mathbb{Z}^{d}$ if the eigenvalues of $A$ satisfy $|\lambda|>1$.

The subband filters $m_{i}$ are defined in terms of this map, $r_{A}$, and the problem is now to realize the wavelet data in the Hilbert space $L^{2}\left(\mathbb{R}^{d}\right)$ in such a way that $r=r_{A}: X \rightarrow X$ induces the unitary scaling operator $f \mapsto N^{1 / 2} f(A x)$ in $L^{2}\left(\mathbb{R}^{d}\right)$; see (2.7).

2.2. Examples (Julia sets, subshifts). In this paper we will show that this extension from spaces $X$, with a finite-to-one mapping $r: X \rightarrow X$, to operator systems may be done quite generally, to apply to the case when $X$ is a Julia set for a fixed rational function of a complex variable, i.e., $r(z)=p_{1}(z) / p_{2}(z)$, with $p_{1}, p_{2}$ polynomials, $z \in \mathbb{C}$ and $N=\max \left(\operatorname{deg} p_{1}, \operatorname{deg} p_{2}\right)$. Then $r: X(r) \rightarrow X(r)$ is $N$-to-1 except at the singular points of $r$. Here $X(r)$ denotes the Julia set of $r$.

It also applies to shift-invariant spaces $X(A)$ when $A$ is a 0-1 matrix and $X(A)=$ $\left\{\left(x_{i}\right) \in \prod_{\mathbb{N}}\{1, \ldots, N\} \mid A\left(x_{i}, x_{i+1}\right)=1\right\}$, while $r_{A}\left(x_{1}, x_{2}, \ldots\right)=\left(x_{2}, x_{3}, \ldots\right)$ is the familiar subshift. Note that $r_{A}: X(A) \rightarrow X(A)$ is onto iff every column in $A$ contains at least one entry 1.

2.3. Martingales. Part of the motivation for our paper derives from the papers by Richard Gundy Gun00, Gun04, Gun99], Gun66]. The second named author also acknowledges enlightening discussions with R. Gundy. The fundamental idea in these papers by Gundy et al. is that multiresolutions should be understood as martingales in the sense of Doob Doob1], Doob2], Doob3] and Neveu [Neveu]. And moreover that this is a natural viewpoint.

One substantial advantage of this viewpoint is that we are then able to handle the construction of wavelets from subband filters that are only assumed measurable, i.e., filters that fail to satisfy the regularity conditions that are traditionally imposed in wavelet analysis.

A second advantage is that the martingale approach applies to a number of wavelet-like constructions completely outside the traditional scope of wavelet analysis in the Hilbert space $L^{2}\left(\mathbb{R}^{d}\right)$. But more importantly, the martingale tools apply even when the operation of scaling does not take place in $\mathbb{R}^{d}$ at all, but rather in a compact Julia set from complex dynamics; or the scaling operation may be one of the shift in the subshift dynamics that is understood from that thermodynamical formalism of David Ruelle [Rue89].

2.4. The general theory. In each of the examples, we are faced with a given space $X$, and a finite-to-one mapping $r: X \rightarrow X$. The space $X$ is equipped with a suitable family of measures $\mu_{h}$, and the $L^{\infty}$ functions on $X$ act by multiplication on the corresponding $L^{2}$ spaces, $L^{2}\left(X, \mu_{h}\right)$. It is easy to see that there are $L^{2}$ isometries which intertwine the multiplication operators $M(g)$ and $M(g \circ r)$, as $g$ ranges over $L^{\infty}(X)$. We have

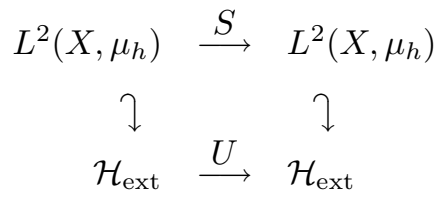


where the vertical maps are given by inclusions. Specifically,

$$
S M(g)=M(g \circ r) S, \text { and } U M(g) U^{-1}=M(g \circ r) .
$$

But for spectral-theoretic calculations, we need to have representations of $M(g)$ and $M(g \circ r)$ that are unitarily equivalent. That is true in traditional wavelet applications, but the unitary operator $U$ in (2.9) is not acting on $L^{2}\left(X, \mu_{h}\right)$. Rather, the unitary $U$ is acting by matrix scaling on a different Hilbert space, namely $L^{2}\left(\mathbb{R}^{d}\right.$, Lebesgue measure), with the scaling operator $U_{A}$ from (2.7).

In the other applications, Julia set, and shift-spaces, we aim for a similar construction. But in these other cases, it is not at all clear what the Hilbert space corresponding to $L^{2}\left(\mathbb{R}^{d}\right)$, and the corresponding unitary matrix scaling operator, should be.

We provide two answers to this question, one at an abstract level, and a second one which is a concrete function representation.

At the abstract level, we show that the construction may be accomplished in Hilbert spaces which serve as unitary dilations of the initial structure; see (2.8).

In the concrete, we show that the extended Hilbert spaces may be taken as Hilbert spaces of $L^{2}$-martingales on $X$. In fact, we present these as Hilbert spaces of certain $L^{2}$-functions on the projective limit $X_{\infty}$ which we outlined in (2.1) above. Only, now the relevant measures are part of this limit construction. This is analogous to the distinction between an abstract spectral theorem on the one hand, and a concrete spectral representation on the other. To know details about multiplicities, and multiplicity functions, we need the latter.

Our concrete version of the dilation Hilbert space $\mathcal{H}_{\text {ext }}$ from $(2.8)$ is then

$$
\mathcal{H}_{\mathrm{ext}} \simeq L^{2}\left(X_{\infty}, \hat{\mu}_{h}\right)
$$

for a suitable measure $\hat{\mu}_{h}$ on $X_{\infty}$.

\section{Functions and Measures on $X$}

Consider $X$ a compact metric space, with $\mathfrak{B}, r, W, \mu$ as follows: $\mathfrak{B}=\mathfrak{B}(X)$ a Borel sigma-algebra of subsets of $X ; r: X \rightarrow X$ an onto, measurable map such that $\# r^{-1}(x)<\infty$ for all $x \in X ; W: X \rightarrow[0, \infty) ; \mu$ a positive Borel measure on $X$.

\subsection{Transformations of functions and measures.}

- Let $g \in L^{\infty}(X)$. Then

$$
M(g) f=g f
$$

is the multiplication operator on $L^{\infty}(X)$ or on $L^{2}(X, \mu)$.

- Composition:

$$
S_{0} f=f \circ r, \text { or }\left(S_{0} f\right)(x)=f(r(x)) \quad(x \in X) .
$$

- If $m_{0} \in L^{\infty}(X)$, we set

$$
S_{m_{0}}=M\left(m_{0}\right) S_{0},
$$

or equivalently

$$
\begin{gathered}
\left(S_{m_{0}} f\right)(x)=m_{0}(x) f(r(x)) \quad\left(x \in X, f \in L^{\infty}(X)\right) . \\
\text { - } r^{-1}(E):=\{x \in X \mid r(x) \in E\} \text { for } E \in \mathfrak{B}(X) \\
\mu \circ r^{-1}(E)=\mu\left(r^{-1}(E)\right) \quad(E \in \mathfrak{B}(X)) .
\end{gathered}
$$




\subsection{Properties of measures $\mu$ on $X$. Definitions.}

(i) Invariance:

$$
\mu \circ r^{-1}=\mu
$$

(ii) Strong invariance:

$$
\int_{X} f(x) d \mu=\int_{X} \frac{1}{\# r^{-1}(x)} \sum_{r(y)=x} f(y) d \mu \quad\left(f \in L^{\infty}(X)\right) .
$$

(iii) $W: X \rightarrow[0, \infty)$,

$$
\left(R_{W} f\right)(x)=\sum_{r(y)=x} W(y) f(y)
$$

If $m_{0} \in L^{\infty}(X, \mu)$ is complex valued, we use the notation $R_{m_{0}}:=R_{W}$ where $W(x)=\left|m_{0}(x)\right|^{2} / \# r^{-1}(r(x))$.

(a) A function $h: X \rightarrow[0, \infty)$ is said to be an eigenfunction for $R_{W}$ if

$$
R_{W} h=h \text {. }
$$

(b) A Borel measure $\nu$ on $X$ is said to be a left eigenfunction for $R_{W}$ if

$$
\nu R_{W}=\nu
$$

or equivalently

$$
\int_{X} R_{W} f d \nu=\int_{X} f d \nu, \text { for all } f \in L^{\infty}(X)
$$

Lemma 3.1. $\quad$ (i) For measures $\mu$ on $X$ we have the implication (3.5) $\Rightarrow$ (3.4), but not conversely.

(ii) If $W$ is given and if $\nu$ and $h$ satisfy (3.8) and (3.7) respectively, then

$$
d \mu:=h d \nu
$$

satisfies (3.4).

(iii) If $\mu$ satisfies (3.5), and $m_{0} \in L^{\infty}(X)$, then $S_{m_{0}}$ is an isometry in $L^{2}(X, h d \mu)$ if and only if

$$
R_{m_{0}} h=h .
$$

3.3. Examples. We illustrate the definitions.

Example 3.2. Let $X=[0,1]=\mathbb{R} / \mathbb{Z}$. Fix $N \in \mathbb{Z}_{+}, N>1$. Let

$$
r(x)=N x \bmod 1 \text {. }
$$

Invariance:

$$
\int_{0}^{1} f(N x) d \mu(x)=\int_{0}^{1} f(x) d \mu(x) \quad\left(f \in L^{\infty}(\mathbb{R} / \mathbb{Z})\right) .
$$

Strong invariance:

$$
\frac{1}{N} \int_{0}^{1} \sum_{k=0}^{N-1} f\left(\frac{x+k}{N}\right) d \mu(x)=\int_{0}^{1} f(x) d \mu(x) .
$$

The Lebesgue measure $\mu=\lambda$ is the unique probability measure on $[0,1]=\mathbb{R} / \mathbb{Z}$ which satisfies (3.11).

Examples of measures $\mu$ on $\mathbb{R} / \mathbb{Z}$ which satisfy (3.10) but not (3.11) are

- $\mu=\delta_{0}$, the Dirac mass at $x=0$; 
- $\mu=\mu_{\mathbf{C}}$, the Cantor middle-third measure on $[0,1]$ (see [DJ03]), i.e., $\mu_{\mathbf{C}}$ is determined by

$-\frac{1}{2} \int\left(f\left(\frac{x}{3}\right)+f\left(\frac{x+2}{3}\right)\right) d \mu_{\mathbf{C}}(x)=\int f(x) d \mu_{\mathbf{C}}(x)$,

$-\mu_{\mathbf{C}}([0,1])=1$,

- $\mu_{\mathbf{C}}$ is supported on the middle-third Cantor set.

Example 3.3. Let $X=[0,1)=\mathbb{R} / \mathbb{Z}, \lambda$ the Lebesgue measure, $X_{\mathbf{C}}$ the middlethird Cantor set, $\mu_{\mathbf{C}}$ the Cantor measure.

$r: X \rightarrow X, r(x)=3 x \bmod 1, r_{\mathbf{C}}=r_{X_{\mathbf{C}}}: X_{\mathbf{C}} \rightarrow X_{\mathbf{C}}$.

Consider the following properties for a Borel probability measure $\mu$ on $\mathbb{R}$ :

$$
\begin{gathered}
\int f d \mu=\frac{1}{3} \int\left(f\left(\frac{x}{3}\right)+f\left(\frac{x+1}{3}\right)+f\left(\frac{x+2}{3}\right)\right) d \mu(x) ; \\
\int f d \mu=\frac{1}{2} \int\left(f\left(\frac{x}{3}\right)+f\left(\frac{x+2}{3}\right)\right) d \mu(x) .
\end{gathered}
$$

Then (3.12) has a unique solution $\mu=\lambda$. Moreover (3.13) has a unique solution, $\mu=\mu_{\mathbf{C}}$, and $\mu_{\mathbf{C}}$ is supported on the Cantor set $X_{\mathbf{C}}$.

Let $\mathbb{R} / \mathbb{Z}=[0,1)$. Then $\# r^{-1}(x)=3$ for all $x \in[0,1)$. If $x=x_{1} / 3+x_{2} / 3^{2}+\cdots$, $x_{i} \in\{0,1,2\}$, is the representation of $x$ in base 3 , then $r(x) \sim\left(x_{2}, x_{3}, \ldots\right)$, and $r^{-1}(x)=\left\{\left(0, x_{1}, x_{2}, \ldots\right),\left(1, x_{1}, x_{2}, \ldots\right),\left(2, x_{1}, x_{2}, \ldots\right)\right\}$.

On the Cantor set $\# r_{\mathbf{C}}^{-1}(x)=2$ for all $x \in X_{\mathbf{C}}$. If $x=x_{1} / 3+x_{2} / 3^{2}+\cdots$, $x_{i} \in\{0,2\}$, is the usual representation of $X_{\mathbf{C}}$ in base 3 , then $r_{\mathbf{C}}(x)=\left(x_{2}, x_{3}, \ldots\right)$ and $X_{\mathbf{C}} \simeq \prod_{\mathbb{N}}\{0,2\}$.

In the representation $\prod_{\mathbb{N}} \mathbb{Z}_{3}$ of $X=[0,1), \mu=\lambda$ is the product (Bernoulli) measure with weights $(1 / 3,1 / 3,1 / 3)$.

In the representation $\prod_{\mathbb{N}}\{0,2\}$ of $X_{\mathbf{C}}, \mu_{\mathbf{C}}$ is the product (Bernoulli) measure with weights $(1 / 2,1 / 2)$.

Example 3.4. Let $N \in \mathbb{Z}_{+}, N \geq 2$, let $A=\left(a_{i j}\right)_{i, j=1}^{N}$ be an $N$ by $N$ matrix with all $a_{i j} \in\{0,1\}$, let $X(A)$ be the corresponding state space, and let $r=r_{A}$.

Lemma 3.5. Let $A$ be as above. Then

$$
\# r_{A}^{-1}(x)=\#\left\{y \in\{1, \ldots, N\} \mid A\left(y, x_{1}\right)=1\right\} .
$$

It follows that $r_{A}: X(A) \rightarrow X(A)$ is onto iff $A$ is irreducible, i.e., iff for all $j \in \mathbb{Z}_{N}$, there exists an $i \in \mathbb{Z}_{N}$ such that $A(i, j)=1$.

The existence of strongly invariant measures is guaranteed by results of Ruelle Rue89 for the case of subshifts of finite type, Brolin [Bro] for rational maps, and Bal00] for a class of expanding maps.

One of the tools from operator theory which has been especially useful in the analysis of wavelets is multiplicity theory for abelian $C^{*}$-algebras $\mathcal{A}$.

We first recall a few well known facts; see, e.g., [N]. By Gelfand's theorem, every abelian $C^{*}$-algebra with unity is $C(X)$ for a compact Hausdorff space $X$; and every representation of $\mathcal{A}$ is the orthogonal sum of cyclic representations. While the cardinality of the set of cyclic components in this decomposition is an invariant, the explicit determination of the cyclic components is problematic, as the construction depends on Zorn's lemma. 
Suppose now that $H$ is a Hilbert space with an isometry $S$ on it and with a normal representation $\pi$ of $L^{\infty}(X)$ on $H$ that satisfies the covariance relation

$$
S \pi(g)=\pi(g \circ r) S \quad\left(g \in L^{\infty}(X)\right) .
$$

Theorem 3.6 shows that there exist a Hilbert space $\hat{H}$ containing $H$, a unitary $\hat{S}$ on $\hat{H}$ and a representation $\hat{\pi}$ of $L^{\infty}(X)$ on $\hat{H}$ such that:

$\left(V_{n}:=\hat{S}^{-n}(H)\right)_{n}$ form an increasing sequence of subspaces with dense union,

$$
\begin{gathered}
\left.\hat{S}\right|_{H}=S, \\
\left.\hat{\pi}\right|_{H}=\pi, \\
\hat{S} \hat{\pi}(g)=\hat{\pi}(g \circ r) \hat{S} .
\end{gathered}
$$

Theorem 3.6. (i) Let $H$ be a Hilbert space, and $S$ an isometry on $H$. Then there exist a Hilbert space $\hat{H}$ containing $H$ and a unitary $\hat{S}$ on $\hat{H}$ such that

$$
\begin{gathered}
\left.\hat{S}\right|_{H}=S, \\
\overline{\bigcup_{n \geq 0} \hat{S}^{-n} H}=\hat{H} .
\end{gathered}
$$

Moreover, these are unique up to an intertwining isomorphism.

(ii) If $\mathcal{A}$ is a $C^{*}$-algebra, $\alpha$ is an endomorphism on $\mathcal{A}$, and $\pi$ is a representation of $\mathcal{A}$ on $H$ such that

$$
S \pi(g)=\pi(\alpha(g)) S \quad(g \in \mathcal{A}),
$$

then there exists a unique representation $\hat{\pi}$ on $\hat{H}$ such that

$$
\left.\hat{\pi}(g)\right|_{H}=\pi(g) \quad(g \in \mathcal{A}),
$$

$$
\hat{S} \hat{\pi}(g)=\hat{\pi}(\alpha(g)) \hat{S} \quad(g \in \mathcal{A}) .
$$

Corollary 3.7. Let $X, r$, and $\mu$ be as above. Let $I$ be a finite or countable set. Suppose $H: X \rightarrow \mathcal{B}\left(l^{2}(I)\right)$ has the property that $H(x) \geq 0$ for almost every $x \in X$, and $H_{i j} \in L^{1}(X)$ for all $i, j \in I$. Let $M_{0}: X \rightarrow \mathcal{B}\left(l^{2}(I)\right)$ such that $x \mapsto\left\|M_{0}(x)\right\|$ is essentially bounded. Assume in addition that

$$
\frac{1}{\# r^{-1}(x)} \sum_{r(y)=x} M_{0}^{*}(y) H(y) M_{0}(y)=H(x), \text { for a.e. } x \in X .
$$

Then there exist a Hilbert space $\hat{K}$, a unitary operator $\hat{U}$ on $\hat{K}$, a representation $\hat{\pi}$ of $L^{\infty}(X)$ on $\hat{K}$, and a family of vectors $\left(\varphi_{i}\right) \in \hat{K}$, such that:

$$
\begin{gathered}
\hat{U} \hat{\pi}(g) \hat{U}^{-1}=\hat{\pi}(g \circ r) \quad\left(g \in L^{\infty}(X)\right), \\
\hat{U} \varphi_{i}=\sum_{j \in I} \hat{\pi}\left(\left(M_{0}\right)_{j i}\right) \varphi_{j} \quad(i \in I), \\
\left\langle\varphi_{i} \mid \hat{\pi}(f) \varphi_{j}\right\rangle=\int_{X} f H_{i j} d \mu \quad\left(i, j \in I, f \in L^{\infty}(X)\right), \\
\overline{\operatorname{span}}\left\{\hat{\pi}(f) \varphi_{i} \mid n \geq 0, f \in L^{\infty}(X), i \in I\right\}=\hat{K} .
\end{gathered}
$$


These are unique up to an intertwining unitary isomorphism. (All functions are assumed weakly measurable in the sense that $x \mapsto\langle\xi \mid F(x) \eta\rangle$ is measurable for all $\xi, \eta \in l^{2}(I)$.)

Note that $\hat{S}$ maps $V_{1}$ to $V_{0}$, and the covariance relation implies that the representation $\hat{\pi}$ on $V_{1}$ is isomorphic to the representation $\pi^{r}: g \mapsto \pi(g \circ r)$ on $V_{0}$. Therefore we have to compute the multiplicity of the latter, which we denote by $d_{V_{0}}^{r}$.

By the spectral theorem there exists a unitary isomorphism $J: H\left(=V_{0}\right) \rightarrow$ $L^{2}\left(X, d_{V_{0}}, \mu\right)$, where, for a multiplicity function $d: X \rightarrow\{0,1, \ldots, \infty\}$, we use the notation:

$$
L^{2}(X, d, \mu):=\left\{f: X \rightarrow \bigcup_{x \in X} \mathbb{C}^{d(x)} \mid f(x) \in \mathbb{C}^{d(x)}, \int_{X}\|f(x)\|^{2} d \mu(x)<\infty\right\} .
$$

In addition $J$ intertwines $\pi$ with the representation of $L^{\infty}(X)$ by multiplication operators, i.e.,

$$
\left(J \pi(g) J^{-1}(f)\right)(x)=g(x) f(x) \quad\left(g \in L^{\infty}(X), f \in L^{2}\left(X, d_{V_{0}}, \mu\right), x \in X\right) .
$$

Remark 3.8. Here we are identifying $H$ with $L^{2}\left(X, d_{V_{0}}, \mu\right)$ via the spectral representation. We recall the details of this representation $H \ni f \mapsto \tilde{f} \in L^{2}\left(X, d_{V_{0}}, \mu\right)$.

Recall that any normal representation $\pi \in \operatorname{Rep}\left(L^{\infty}(X), H\right)$ is the orthogonal sum

$$
H=\sum_{k \in C}^{\oplus}\left[\pi\left(L^{\infty}(X)\right) k\right],
$$

where the set $C$ of vectors $k \in H$ is chosen such that

$$
\begin{gathered}
\|k\|=1, \\
\langle k \mid \pi(g) k\rangle=\int_{X} g(x) v_{k}(x)^{2} d \mu(x), \text { for all } k \in C \\
\text { - }\left\langle k^{\prime} \mid \pi(g) k\right\rangle=0, \quad g \in L^{\infty}(X), k, k^{\prime} \in C, k \neq k^{\prime} \text {; orthogonality. }
\end{gathered}
$$

Formula (3.21) is obtained by a use of Zorn's lemma. Here, $v_{k}^{2}$ is the RadonNikodym derivative of $\langle k \mid \pi(\cdot) k\rangle$ with respect to $\mu$, and we use that $\pi$ is assumed normal.

For $f \in H$, set

$$
f=\sum_{k \in C}^{\oplus} \pi\left(g_{k}\right) k, \quad g_{k} \in L^{\infty}(X)
$$

and

$$
\tilde{f}=\sum_{k \in C}^{\oplus} g_{k} v_{k} \in L_{\mu}^{2}\left(X, l^{2}(C)\right) .
$$

Then $W f=\tilde{f}$ is the desired spectral transform, i.e.,

$$
W \text { is unitary, }
$$

$$
W \pi(g)=M(g) W
$$

and

Indeed, we have

$$
\|\tilde{f}(x)\|^{2}=\sum_{k \in C}\left|g_{k}(x) v_{k}(x)\right|^{2}
$$

$$
\int_{X}\|\tilde{f}(x)\|^{2} d \mu(x)=\int_{X} \sum_{k \in C}\left|g_{k}(x)\right|^{2} v_{k}(x)^{2} d \mu(x)=\sum_{k \in C} \int_{X}\left|g_{k}\right|^{2} v_{k}^{2} d \mu .
$$




$$
=\sum_{k \in C}\left\langle k \mid \pi\left(\left|g_{k}\right|^{2}\right) k\right\rangle=\sum_{k \in C}\left\|\pi\left(g_{k}\right) k\right\|^{2}=\left\|\sum_{k \in C}^{\oplus} \pi\left(g_{k}\right) k\right\|_{H}^{2}=\|f\|_{H}^{2} .
$$

It follows in particular that the multiplicity function $d(x)=d_{H}(x)$ is

$$
d(x)=\#\left\{k \in C \mid v_{k}(x) \neq 0\right\} .
$$

Setting

we see that

$$
X_{i}:=\{x \in X \mid d(x) \geq i\} \quad(i \geq 1)
$$

$$
H \simeq \sum^{\oplus} L^{2}\left(X_{i}, \mu\right) \simeq L^{2}(X, d, \mu)
$$

and the isomorphism intertwines $\pi(g)$ with multiplication operators.

Theorem 3.9. (i) $V_{1}=\hat{S}^{-1}(H)$ is invariant for the representation $\hat{\pi}$. The multiplicity functions of the representation $\hat{\pi}$ on $V_{1}$, and on $V_{0}=H$, are related by

$$
d_{V_{1}}(x)=\sum_{r(y)=x} d_{V_{0}}(y) \quad(x \in X) .
$$

(ii) If $W_{0}:=V_{1} \ominus V_{0}=\hat{S}^{-1} H \ominus H$, then

$$
d_{V_{0}}(x)+d_{W_{0}}(x)=\sum_{r(y)=x} d_{V_{0}}(y) \quad(x \in X) .
$$

With the spaces $H_{n}$ in (3.26), our global Hilbert space of discrete $L^{2}$-martingales thus acquires a concrete form, and we are now able to present our multiresolution/wavelet result below.

Theorem 3.10. If $h \in L^{1}(X), h \geq 0$, and $R h=h$, then there exists a unique measure $\hat{\mu}$ on $X_{\infty}$ such that

$$
\hat{\mu} \circ \theta_{n}^{-1}=\omega_{n} \quad(n \geq 0),
$$

where

$$
\omega_{n}(f)=\int_{X} R^{n}(f h) d \mu \quad\left(f \in L^{\infty}(X)\right) .
$$

We now give a different representation of the construction of the covariant system associated to $m_{0}$ and $h$.

Let

$$
H_{n}:=\left\{f \in L^{2}\left(X_{\infty}, \hat{\mu}\right) \mid f=\xi \circ \theta_{n}, \xi \in L^{2}\left(X, \omega_{n}\right)\right\} .
$$

Then $H_{n}$ form an increasing sequence of closed subspaces which have dense union.

We can identify the functions in $H_{n}$ with functions in $L^{2}\left(X, \omega_{n}\right)$, by

$$
i_{n}(\xi)=\xi \circ \theta_{n} \quad\left(\xi \in L^{2}\left(X, \omega_{n}\right)\right) .
$$

The definition of $\hat{\mu}$ makes $i_{n}$ an isomorphism between $H_{n}$ and $L^{2}\left(X, \omega_{n}\right)$.

Define

$\mathcal{H}:=\left\{\left(\xi_{0}, \xi_{1}, \ldots\right) \mid \xi_{n} \in L^{2}\left(X, \omega_{n}\right), R\left(\xi_{n+1} h\right)=\xi_{n} h, \sup _{n} \int_{X} R^{n}\left(\left|\xi_{n}\right|^{2} h\right) d \mu<\infty\right\}$,

with the scalar product

$$
\left\langle\left(\xi_{0}, \xi_{1}, \ldots\right) \mid\left(\eta_{0}, \eta_{1}, \ldots\right)\right\rangle=\lim _{n \rightarrow \infty} \int_{X} R^{n}\left(\bar{\xi}_{n} \eta_{n} h\right) d \mu .
$$


Theorem 3.11. The map $\Phi: L^{2}\left(X_{\infty}, \hat{\mu}\right) \rightarrow \mathcal{H}$ defined by

$$
\Phi(f)=\left(i_{n}^{-1}\left(P_{n} f\right)\right)_{n \geq 0},
$$

where $P_{n}$ is the projection onto $H_{n}$, is an isomorphism. Moreover, this transform $\Phi$ has the following three properties, and it is determined by them:

$$
\begin{gathered}
\Phi U \Phi^{-1}\left(\xi_{n}\right)_{n \geq 0}=\left(m_{0} \circ r^{n} \xi_{n+1}\right)_{n \geq 0}, \\
\Phi \pi(g) \Phi^{-1}\left(\xi_{n}\right)_{n \geq 0}=\left(g \circ r^{n} \xi_{n}\right)_{n \geq 0}, \\
\Phi \varphi=(1,1, \ldots) .
\end{gathered}
$$

Acknowledgements. The authors thank Dick Gundy for helpful discussions, and the National Science Foundation for partial support.

\section{REFERENCES}

[BaM99] L.W. Baggett, K.D. Merrill, Abstract harmonic analysis and wavelets in $\mathbf{R}^{n}$, The Functional and Harmonic Analysis of Wavelets and Frames (San Antonio, 1999) (L.W. Baggett, D.R. Larson, eds.), Contemp. Math., vol. 247, American Mathematical Society, Providence, 1999, pp. 17-27. MR1735967|(2001b:42043)

[Bal00] V. Baladi, Positive Transfer Operators and Decay of Correlations, World Scientific, River Edge, NJ, 2000. MR1793194 (2001k:37035)

[BDP04] S. Bildea, D. Dutkay, G. Picioroaga, MRA Super-wavelets, preprint 2004.

[BK93] M. Bramson, S. Kalikow, Nonuniqueness in g-functions, Israel J. Math. 84 (1993), 153160. MR,1244665 (94h:28011)

[Bro] H. Brolin, Invariant sets under iteration of rational functions, Ark. Mat. 6 (1965), 103144. MR.0194595 (33:2805)

[Dau92] I. Daubechies, Ten Lectures on Wavelets, CBMS-NSF Regional Conf. Ser. in Appl. Math., vol. 61, SIAM, Philadelphia, 1992.

[Doob1] J.L. Doob, The Brownian movement and stochastic equations, Ann. of Math. (2) 43 (1942), 351-369. MR0006634 (4:17d)

[Doob2] , What is a martingale? Amer. Math. Monthly 78 (1971), 451-463. MR0283864 (44:1094)

[Doob3] Classical Potential Theory and Its Probabilistic Counterpart, Grundlehren 262, Springer-Verlag, New York, 1984. MR0731258 (85k:31001)

[DJ03] D.E. Dutkay, P.E.T. Jorgensen, Wavelets on fractals, preprint, University of Iowa, 2003, http://arXiv.org/abs/math.CA/0305443, to appear in Rev. Mat. Iberoamericana.

[DJ04a] Martingales, endomorphisms, and covariant systems of operators in Hilbert space, preprint, University of Iowa, 2004, http://arXiv.org/abs/math.CA/0407330 .

[DJ04b] Operators, martingales, and measures on projective limit spaces, preprint, University of Iowa, 2004, http://arxiv.org/abs/math.CA/0407517.

[DJ04c] _ Disintegration of projective measures, preprint, University of Iowa, 2004, http://arxiv.org/abs/math.CA/0408151 .

[Gun00] R.F. Gundy, Low-pass filters, martingales, and multiresolution analyses, Appl. Comput. Harmon. Anal. 9 (2000), 204-219. MR1777126 (2002k:94013)

[Gun04] _ Wavelets and probability, preprint, Rutgers University, material presented during the author's lecture at the workshop "Wavelets and Applications", Barcelona, Spain, July 1-6, 2002, http://www.imub.ub.es/wavelets/Gundy.pdf .

[Gun66] - Martingale theory and pointwise convergence of certain orthogonal series, Trans. Amer. Math. Soc. 124 (1966), 228-248. MR0204967 (34:4802)

[Gun99] - Two remarks concerning wavelets: Cohen's criterion for low-pass filters and Meyer's theorem on linear independence, The Functional and Harmonic Analysis of Wavelets and Frames (San Antonio, 1999) (L.W. Baggett, D.R. Larson, eds.), Contemp. Math., vol. 247, American Mathematical Society, Providence, 1999, pp. 249-258. MR.1738093 (2001b:42051)

[N] E. Nelson, Topics in Dynamics I: Flows, Mathematical Notes, Princeton University Press, Princeton, NJ, 1969. MR0282379 (43:8091) 
[Neveu] J. Neveu, Discrete-Parameter Martingales, North-Holland, Amsterdam-Oxford, 1975. MR 0402915 (53:6729)

[Rue89] D. Ruelle, The thermodynamic formalism for expanding maps, Comm. Math. Phys. 125 (1989), 239-262. MR1016871 (91a:58149)

Department of Mathematics, The University of Iowa, 14 Maclean Hall, Iowa City, IA $52242-1419$

E-mail address: ddutkay@math.rutgers.edu

Department of Mathematics, The University of Iowa, 14 Maclean Hall, Iowa City, IA $52242-1419$

E-mail address: jorgen@math.uiowa.edu 